\title{
PROCESSAMENTO AUDITIVO, RESOLUÇÃO TEMPORAL E TESTE DE DETECÇÃO DE GAP: REVISÃO DA LITERATURA
}

\author{
Auditory processing, temporal resolution and gap detection test: \\ literature review
}

\author{
Alessandra Giannella Samelli ${ }^{(1)}$, Eliane Schochat ${ }^{(2)}$
}

\begin{abstract}
RESUMO
Tema: processamento auditivo temporal e resolução temporal. Objetivo: realizar revisão teórica sobre processamento auditivo e resolução temporal, bem como sobre os diferentes parâmetros de marcadores utilizados em testes de detecção de gap e como eles podem interferir na determinação dos limiares. Conclusão: o processamento auditivo e a resolução temporal são fundamentais para o desenvolvimento da linguagem. Em virtude dos diferentes parâmetros que podem ser utilizados no teste em questão, os limiares de detecção de gap podem variar consideravelmente.
\end{abstract}

DESCRITORES: Percepção Auditiva; Psico-Acústica; Estimulação Acústica

\section{INTRODUÇÃO}

O tempo é uma dimensão muito importante para a audição, já que quase todos os sons variam ao longo do tempo ${ }^{1}$. Por definição, os sons são eventos físicos (flutuações rápidas de pressão) que estão distribuídos no tempo ${ }^{2}$. Uma citação feita por Lashley, em 1960, afirmava ser o processamento temporal o mais importante e também o mais neglicenciado problema da fisiologia. Quase meio século depois desta afirmação, o estudo do processamento temporal está no início e muitos aspectos sobre o assunto não foram ainda esclarecidos, principalmente, no que diz respeito aos mecanismos neurais que delineiam a percepção sensorial do tempo ${ }^{3}$.

O objetivo desta pesquisa é realizar revisão teórica sobre processamento auditivo e resolução temporal, bem como sobre os diferentes parâmetros de marcadores utilizados em testes de detecção de gap e como eles podem interferir na determinação dos limiares.

(1) Fonoaudióloga; Professora Doutora do Curso de Fonoaudiologia da Faculdade de Medicina da Universidade de São Paulo; Doutora em Ciências pela Faculdade de Medicina da Universidade de São Paulo.

(2) Fonoaudióloga; Professora Associada Doutora do Curso de Fonoaudiologia da Faculdade de Medicina da Universidade de São Paulo; Livre Docente pela Faculdade de Medicina da Universidade de São Paulo.

\section{MÉTODOS}

Foi realizada pesquisa bibliográfica em periódicos nacionais e internacionais, livros e documentos eletrônicos, considerando-se a relevância de cada documento para o assunto em questão.

Inicialmente, foram consultados documentos pelas bases de dados MEDLINE e LILACS, por meio dos descritores auditory processing, temporal resolution e gap detection threshold, sem restrição de data.

$\mathrm{Na}$ medida em que a pesquisa prosseguia, referências bibliográficas importantes, citadas nos documentos já pesquisados, também foram consultadas.

O estudo foi desenvolvido considerando-se os seguintes tópicos: processamento auditivo temporal (ordenação, integração, mascaramento, resolução), resolução temporal e teste de detecção de gap (todas as variáveis que podem ser modificadas nos testes).

\section{REVISÃO DA LITERATURA}

\section{Processamento auditivo temporal}

O processamento auditivo temporal pode ser definido como a percepção do som ou da alteração do som dentro de um período restrito e definido de tempo, ou seja, refere-se à habilidade de perceber 
ou diferenciar estímulos que são apresentados numa rápida sucessão ${ }^{4-6}$.

Muitas evidências sugerem que as habilidades do processamento temporal são a base do processamento auditivo, especificamente no que concerne à percepção de fala. $\mathrm{O}$ argumento que suporta esta proposição é que muitas características da informação auditiva são, de alguma forma, influenciadas pelo tempo ${ }^{4,6,7}$.

A codificação sensorial da informação temporal como duração, intervalo e ordem de diferentes padrões de estímulo provê informações vitais para o sistema nervoso. Todas estas pistas, que regem o processamento temporal, são importantes para a percepção da fala e da música, uma vez que a estrutura destes dois eventos apresenta-se como rápidas mudanças do sinal acústico ${ }^{3,6,8}$.

A identificação de sílabas consoante-vogal individuais, por exemplo, está relacionada com o intervalo entre a liberação do ar e a vibração das pregas vocais (/ba/ versus /pa/); e com o tempo de silêncio entre as consoantes e as vogais (/sa/ versus /sta/). O arranjo seqüencial das sílabas também é importante para o reconhecimento de fala (Ex: la-dy versus de-lay ou sa-co versus co-ça). Da mesma forma, a duração de cada sílaba é crítica, bem como o intervalo entre as sílabas (Ex: "kiss the sky" versus "kiss this guy" ou "Não feche a porta!" versus "Não, feche a porta!" ou "em tão pouco tempo..." versus "então, pouco tempo..."). Além disso, pistas prosódicas como pausas e velocidade de fala são usadas para determinar o conteúdo semântico ${ }^{1,3,6}$.

É importante comentar também que a estrutura de tempo sonora pode ser dividida em eventos periódicos e eventos transitórios. Eventos periódicos (ou repetitivos) são eventos acústicos simples, caracterizados por padrões estáveis. Esta estrutura acústica periódica pode ser expressa em termos de freqüência e fase. Estes eventos periódicos podem gerar a percepção de pitch. É o caso, por exemplo, do pitch da voz humana. Esta sensação subjetiva de freqüência é determinada em grande parte pela taxa de pulsos glóticos. Cada pulso é um sinal relativamente ruidoso, mas o pitch da voz é modelado pela taxa de repetição glótica (freqüência temporal) e pelo conteúdo espectral do trato vocal ${ }^{1,2,9}$.

No entanto, a riqueza do processamento auditivo temporal, isto é, a percepção de mudanças rápidas ao longo do tempo, tem como base a estrutura dos eventos transitórios. Eventos acústicos transitórios (ou aperiódicos) podem ser definidos como eventos acústicos breves, que devem ser segregados e percebidos separadamente de outros eventos acústi$\cos ^{1,2,9}$.

Pode-se ter uma idéia da importância dos eventos transitórios para a percepção da fala, anali- sando-se o tempo de início da sonorização (voice onset time - VOT). No caso da discriminação entre consoantes plosivas surdas e sonoras (Ex: /pa/ versus /ba/), uma das maiores pistas perceptuais é dada pelo VOT, ou seja, o curto tempo entre a "explosão" (consoante - evento transitório) e o início da emissão (vogal - evento periódico) ${ }^{2,9,10}$.

Os VOTs mais curtos (até $30 \mathrm{~ms}$ ) são percebidos como consoantes sonoras, enquanto os VOTs mais longos (de 30 a $60 \mathrm{~ms}$ ) são percebidos como consoantes surdas $5,6,10$.

O processamento auditivo temporal pode ser dividido em quatro categorias, sendo todas importantes para as habilidades de processamento auditivo. São elas ${ }^{4,11,12}$.

1.1 Ordenação ou seqüencialização temporal;

1.2 Integração ou somação temporal;

1.3 Mascaramento temporal;

1.4 Resolução, discriminação ou acuidade temporal.

\subsection{Ordenação ou seqüencialização temporal}

A habilidade auditiva de ordenação temporal refere-se ao processamento de múltiplos estímulos auditivos na sua ordem de ocorrência. Graças a esta habilidade, um indivíduo é capaz de discriminar a correta ordem de ocorrência dos sons. $\mathrm{Na}$ prática clínica, a ordenação temporal é freqüentemente avaliada por meio dos testes de padrão de freqüência e duração, nos quais os pacientes devem verbalizar a ordem da seqüência de três tons ouvida. Para o teste de padrão de duração, um mesmo tom é mantido $(1000 \mathrm{~Hz})$ e a duração é o aspecto a ser discriminado e ordenado. Os estímulos curtos possuem $250 \mathrm{~ms}$, enquanto os longos, $500 \mathrm{~ms}$. O intervalo entre os tons é de $300 \mathrm{~ms}$. Já para o teste de padrão de freqüência, a duração é mantida e utilizam-se seqüências compostas por tons de 880 e $1122 \mathrm{~Hz}$, com 150 ms de separação entre eles ${ }^{4,12-14}$.

\subsection{Integração ou somação temporal}

Em experimentos de integração temporal, os sujeitos devem detectar sinais fracos em um ruído de fundo ou no silêncio. O limiar deste sinal fraco é medido em função de sua duração. Geralmente, a detecção do sinal é a mesma se o produto da duração e da intensidade do sinal se mantiver constante (energia constante) em pelo menos algumas escalas de duração. Para durações excedendo 500 ms, aproximadamente, a intensidade do som no limiar é independente da duração. Contudo, para durações menores que $200 \mathrm{~ms}$, aproximadamente, a intensidade necessária para detecção aumenta com a diminuição da duração. Além disso, em uma dada intensidade, a loudness aumenta com o aumento 
da duração de 100 a 200 ms. O limiar de detecção melhora com o aumento da duração do sinal entre 200 e 300 ms, numa população normal. Se um som for diminuído em 1/10 de sua duração original, o limiar do sujeito piora em aproximadamente 10 $\mathrm{dB}$. A habilidade de integração temporal decorre da somação da atividade neuronal, resultante de uma adicional duração da energia sonora $1,4,13,14$.

\subsection{Mascaramento temporal}

O mascaramento temporal é caracterizado pela mudança do limiar de um som na presença de outro estímulo subseqüente. Isto ocorre quando um estímulo é apresentado com duração e intensidade suficientes para reduzir a sensibilidade de outro estímulo apresentado antes ou depois do estímulo inicial. Sinais curtos são apresentados com diferentes intervalos de tempo, em relação ao som mascarador. Se este sinal preceder o mascarador, a tarefa é chamada de "mascaramento sucessivo" (backward masking); se o sinal seguir o mascarador, o processo é o "mascaramento antecessor" (forward masking) ${ }^{1,4,5}$.

Alguns estudos sugeriram que o "mascaramento antecessor" pode ocorrer em intervalos acima de 75 $\mathrm{ms}$, enquanto o "mascaramento sucessor" é efetivo com intervalos acima de $50 \mathrm{~ms}$. O processo para o "mascaramento sucessivo" não é totalmente compreendido, apesar de extensamente estudado. No que se refere ao "mascaramento antecessor", a influência do mascarador sobre o sinal decai a zero depois de 100 a $200 \mathrm{~ms}$. A base deste tipo de mascaramento também não é completamente entendida, mas a resposta da membrana basilar ao mascarador, que continua por algum tempo após o término do estímulo (ringing ou reverberação) deve contribuir para este efeito. A reverberação se sobrepõe à resposta do sinal e contribui, então, para o mascaramento do sinal ${ }^{1,4,5}$.

\subsection{Resolução, discriminação ou acuidade temporal}

A habilidade auditiva de resolução temporal refere-se ao mínimo tempo requerido para segregar ou resolver eventos acústicos. O limiar para resolução temporal é conhecido como acuidade auditiva ou tempo mínimo de integração temporal ${ }^{4,5,15}$.

Muitos padrões que distinguem os sons da fala baseiam-se em diferenças temporais de poucos milissegundos. Este aspecto do funcionamento do sistema auditivo, no qual mudanças acústicas transitórias podem ser acuradamente identificadas, é fundamental para a compreensão da fala humana, constituindo-se num pré-requisito para as habilidades lingüísticas, bem como para a leitura ${ }^{5,15,16}$.
O VOT é um exemplo da importância da resolução temporal para a fala. A distinção perceptual entre dois fonemas (surdo / sonoro. Ex: /pa/ versus /ba/) é largamente baseada no VOT, o comprimento do intervalo de silêncio entre a explosão de ruído (consoante) e a vogal seguinte. O limiar perceptual, neste caso, é de $35 \mathrm{~ms}^{5,6,16}$.

\section{Teste de Detecção de Gap}

O teste denominado deteç̧ão de gap é um método psicoacústico relativamente simples que mede a resolução temporal ${ }^{9,17-19}$.

Na maioria dos estudos, são apresentados para os sujeitos dois estímulos acústicos (ruído ou tom puro) longos (centenas de milissegundos). Um dos estímulos contém um breve (poucos milissegundos) período de silêncio - o gap - na sua porção medial e o outro estímulo não possui nenhum gap. A tarefa do sujeito consiste em indicar quais estímulos possuem o gap. Normalmente, são utilizados intervalos de gap variados e o limiar de detecção do gap repetitivo consiste no menor intervalo de silêncio percebido pelo sujeito ${ }^{9,14,16}$.

O teste de detecção de gap ${ }^{9,17-19}$ é baseado no paradigma que buscava estudar a taxa de decay da sensação auditiva. Foi investigado o intervalo mínimo de silêncio a ser introduzido entre dois sons (ruído branco) para ser percebido. Durante a pesquisa, foram usados diferentes níveis de intensidade para os dois estímulos. Como resultados, foram obtidos limiares entre 2 e $3 \mathrm{~ms}$, variando de acordo com os níveis de intensidade ${ }^{20}$. Com base neste estudo, foi sugerido que a resolução temporal é limitada pelo decay da sensação auditiva produzida pela primeira parte do estímulo, que preencheria o gap ${ }^{21}$.

Embora o estudo citado anteriormente ${ }^{20}$ tenha utilizado estímulos compostos por ruído branco, atualmente existem diversos estímulos e modos de apresentação aplicados para o estudo do gap. Dada a variabilidade de limiares de gap possíveis resultantes dos diversos procedimentos empregados, é necessário que estes efeitos sejam comentados.

\subsection{Marcadores}

$\mathrm{Na}$ maioria dos estudos, os marcadores são compostos por tons puros e por ruídos de banda. São denominados marcadores, os estímulos acústicos que delimitam os intervalos de silêncio (gaps).

Muitas investigações foram feitas para estudar os efeitos das freqüências dos marcadores sobre o gap ${ }^{1,9,17}$. Os diversos resultados são concordantes, na medida em que demonstraram que, quanto maior a separação de freqüências entre os dois marcadores, maior a deterioração do gap, ou seja, pior o limiar de detecção. 
Em um estudo sobre o assunto, os estímulos empregados foram tons harmônicos complexos de freqüências fundamentais de 140 e $350 \mathrm{~Hz}$. Quando ambos os marcadores possuíam a mesma freqüência fundamental (140 ou $350 \mathrm{~Hz}$ ), a média foi de 5,7 e de $2,4 \mathrm{~ms}$, respectivamente. No entanto, quando as freqüências fundamentais diferiram entre os dois marcadores (140-350 Hz ou 350-140 Hz), a média dos limiares para as duas condições foi de 13,2 ms, isto é, houve um efeito negativo sobre a detecção do gap ${ }^{17}$.

Outro aspecto que pôde ser observado no estudo supracitado ${ }^{17}$ foi a diferença entre as médias dos limiares para as duas freqüências fundamentais. Para a freqüência mais baixa $(140 \mathrm{~Hz})$, obtevese uma média de limiar mais alta, enquanto para $350 \mathrm{~Hz}$, a média foi melhor.

Este fato, ou seja, limiares de detecção melhores para freqüências mais altas, também foi investigado por muitos autores, e este aspecto já é uma característica amplamente aceita nos estudos sobre o assunto.

Numa discussão sobre o tema, ou seja, sobre uma pior resolução temporal para as freqüências mais baixas, foi sugerido que a explicação estava no tempo de resposta dos filtros auditivos. Quanto mais estreita a largura de banda de um filtro, mais longo é seu tempo de resposta. Uma vez que os filtros no sistema auditivo periférico possuem largura de banda mais estreita, nas freqüências mais baixas do que nas altas, então, as respostas para as freqüências mais baixas são mais longas. Desta forma, a reverberação (ringing) para as freqüências mais baixas preenche por mais tempo o gap, limitando mais a sua resolução temporal 1,22,23.

Com relação aos marcadores compostos por ruído, os ruídos de banda possuem flutuações inerentes na amplitude. Assim, os limiares de gap para ruídos de banda devem ser parcialmente limitados pelas flutuações do ruído. Os declives que ocorrem no ruído podem ser confundidos com os gaps. Por este motivo, os marcadores de ruído de banda estreita (percepção do som altamente flutuante) dão origem a limiares de gap mais pobres, enquanto ruído de banda larga (percepção mais suave das flutuações) resulta em limiares de gap melhores. Os limiares de gap melhores, obtidos com ruído de banda larga refletem o uso da informação das regiões de alta freqüência do espectro, uma vez que os limiares de gap para freqüências mais altas são melhores que os limiares para freqüências mais baixas ${ }^{1,5,15}$.

\subsection{Intensidade dos marcadores}

Com relação à intensidade dos marcadores, em um estudo sobre o assunto, esta foi variada de 10 dB NS a 75 dB NS, obtendo-se gaps entre 2 e 25 ms.
Os menores limiares resultaram de marcadores apresentados em intensidades mais elevadas 1,20.

Muitos outros estudos também demonstraram esta relação entre nível de apresentação dos marcadores e limiar de detecção do gap. Para ruídos de banda larga, o limiar de gap aumenta para níveis de estimulação mais baixos, próximos ao limiar absoluto, mas é relativamente invariante com intensidades moderadas a elevadas. O mesmo padrão é observado para ruídos de banda estreita; o limiar de gap tende a diminuir com o aumento dos níveis sonoros até aproximadamente $30 \mathrm{~dB}$ NS, mas permanece constante depois disso ${ }^{1,17,20}$.

\subsection{Duração dos marcadores}

Segundo uma investigação feita sobre o assunto, a performance dos testes para detecção de gaps é significantemente melhor para marcadores mais longos. Foram usados ruídos com filtro passa-baixo (corte em $5 \mathrm{kHz}$ ) de 100 a $400 \mathrm{~ms}$. Os limiares para adultos jovens foram 4,14 ms para o marcador de $100 \mathrm{~ms}$ e $3,46 \mathrm{~ms}$ para o marcador de $400 \mathrm{~ms}$. A explicação dada para este achado foi a influência da loudness que aumenta com o aumento da duração do estímulo (integração temporal). Uma vez que a tarefa de detecção de gap requer tanto a habilidade de resolução temporal como a de resolução de intensidade, houve uma correlação significante entre detecção do gap e influência da duração do estímulo, paralela à influência de intensidade ${ }^{21}$.

Outro paradigma que pode ser estudado, com base na duração dos marcadores, é a variação do primeiro marcador ou do segundo marcador, enquanto um deles se mantém fixo.

Este paradigma foi investigado em um trabalho, no qual o primeiro marcador variou entre 5 e 300 $\mathrm{ms}$, enquanto o segundo marcador foi fixado em 300 ms. Os autores observaram um aumento dos limiares de gap quando o primeiro marcador era mais curto, mas apenas quando utilizaram ruídos diferentes entre os dois marcadores (ruído centrado em 1 e $4 \mathrm{kHz}$ ). Para as condições de teste nas quais os dois marcadores eram ruídos iguais (ruído centrado em 1 ou 4 kHz), não houve influência da duração do primeiro marcador nos limiares de gap ${ }^{9}$.

Não existe uma concordância geral, no que se refere à duração dos marcadores na determinação dos limiares de gap. Influências como freqüência, intensidade e a própria duração dos marcadores devem ser levadas em consideração, bem como a participação dos mecanismos de integração temporal e mascaramento temporal ${ }^{1,9,21}$.

\subsection{Posição do gap dentro dos marcadores}

A duração dos marcadores possui uma relação direta com o posicionamento do gap dentro dos 
mesmos. Quanto mais curto for o primeiro marcador, mais inicial será a posição do gap a ser detectado.

Os paradigmas convencionais de detecção de gap colocavam os gaps usualmente fixos no centro dos marcadores. Contudo, dada a importância da detecção dos eventos transitórios para a resolução temporal, alguns autores estudaram a colocação randômica dos gaps dentro dos marcadores. Este fato buscou aproximar o teste de detecção do gap da estrutura normal de fala, na qual os intervalos de silêncio ocorrem em diferentes posições dentro da fala contínua ${ }^{9,10,21}$

Um estudo neurofisiológico do córtex auditivo revelou que a codificação dos gaps é mais pobre para gaps que ocorrem precocemente (5 ms após o início do som) quando comparados com gaps posicionados mais tardiamente (500 ms após o início do som). Os gaps foram colocados em um ruído de banda larga de um segundo de duração e a atividade neural de áreas auditivas corticais foi medida. $\mathrm{O}$ autor comparou os gaps precoces (5 ms após o início do ruído) aos VOTs das sílabas /ba/ e /pa/ e observou que os neurônios corticais responderam para estes gaps somente quando eram maiores do que $30 \mathrm{~ms}$. Já para os gaps tardios (500 ms após o início do ruído), respostas neurais foram obtidas para gaps repetitivo tão curtos como $5 \mathrm{~ms}^{10}$.

Por meio de marcadores de ruído de banda larga e de banda estreita, a posição do gap dentro dos marcadores foi variada em: 5, 10, 30, 50, 100 e 300 $\mathrm{ms}$. Os autores constataram que para gaps inseridos precocemente houve uma piora dos limiares de detecção de gap. Para os gaps precoces, a média do limiar de gap foi 32,4 ms, enquanto para os gaps tardios, a média foi próxima de $10 \mathrm{~ms}^{9}$.

A conclusão deste experimento ${ }^{9}$ foi semelhante à do estudo anterior ${ }^{10}$, ou seja, para os gaps precoces (semelhantes ao VOT), os limiares de gap foram aproximadamente $30 \mathrm{~ms}$. Este limiar é muito próximo aos valores definidos como sendo fundamentais para discriminação entre consoantes surdas e sonoras, ou seja, até $30 \mathrm{~ms}^{10,21}$.

\subsection{Apresentação dos estímulos} (monoaural versus binaural)

A avaliação da percepção do gap pode ser monoaural ou binaural. A condição binaural pode ser estabelecida por meio de fones ou campo livre.

Foram utilizados os paradigmas de teste binaural versus monoaural. O primeiro marcador foi dado sempre na orelha esquerda e o segundo marcador era apresentado ora na orelha esquerda, ora na orelha direita e ora em ambas as orelhas simultaneamente. Os limiares de gap para as condições esquerda-esquerda e esquerda-ambas foram os mais baixos (aproximadamente 4 a $5 \mathrm{~ms}$ ). Entre- tanto, na condição esquerda-direita, os limiares foram muito mais altos (17 a $32 \mathrm{~ms}$, dependendo da duração do estímulo) ${ }^{9}$.

Da mesma forma, o efeito do modo de apresentação sobre o limiar de gap foi avaliado. Alto-falantes suspensos na altura das orelhas foram usados. As condições de teste foram: esquerda-esquerda, direita-direita, esquerda-direita e direita-esquerda. Nas condições binaurais, os marcadores foram idênticos nas duas orelhas (diótico), ou possuíam diferenças interaurais de intensidade $(12 \mathrm{~dB})$ ou de tempo (640 ms); estas diferenças foram suficientes para lateralizar o estímulo para um ou outro lado. Como resultados, os limiares de gap não foram afetados pela diferença de tempo interaural, sugerindo que uma mudança na localização percebida não necessariamente eleva os limiares de gap. Além disso, embora as diferenças interaurais de intensidade tenham interferido pronunciadamente nos limiares de gap, a mesma deterioração foi produzida por uma diferença monoaural de intensidade ${ }^{17}$.

Sendo assim, o autor ${ }^{17}$ concluiu que o sistema binaural tem um papel pequeno ou inexistente nestas tarefas de detecção de gap e que os limiares em todas as condições podem ser entendidos unicamente como resultado dos sinais apresentados para cada orelha separadamente. Acrescentou, ainda, que o efeito espacial sobre o gap encontrado em estudos anteriores seria conseqüência das diferenças de intensidade monoaurais introduzidas em cada orelha.

Por isso, este autor ${ }^{17}$ resumiu seus achados afirmando que, se a informação monoaural está disponível, nestas tarefas de detecção de gap, a performance dos ouvintes não pode ser afetada pela informação binaural.

\subsection{Efeito do tempo de surgimento do sinal} (rise) e tempo de declínio do sinal (fall)

Um dos maiores problemas técnicos encontrados em estudos de resolução temporal é a dificuldade de gerar dois estímulos auditivos que difiram apenas nos seus aspectos temporais. Freqüentemente, alterações temporais na onda sonora produzem outras pistas indesejáveis como, por exemplo, as pistas espectrais. Estas variações espectrais tornam-se, então, a base para a tarefa temporal, ao invés das próprias pistas temporais ${ }^{1,5,15}$.

No caso de tons puros, o espectro do início do tom contém um pico na freqüência específica do tom e tempos de início e declínio que se estendem, com amplitude decrescente, simetricamente, para cima e para baixo desta freqüência. A amplitude e largura dos tempos de surgimento e declínio do som variam inversamente com o tempo de início do estímulo ${ }^{1,5,15}$. 
No caso de pesquisas psicofísicas com humanos, observou-se que os limiares de detecção para sinais tonais são mais baixos em estímulos de rápido início. Além disso, a percepção da loudness aumenta para tons de amplitude equivalente, mas com tempo de início mais rápido. Estes dois efeitos são conseqüências da "agitação" espectral (spectral splatter) causada pelo início abrupto do sinal acústico ${ }^{1,5,15}$.

Além disso, se um ruído for adicionado para mascarar estas pistas espectrais, o limiar de detecção do gap aumenta dramaticamente e os resultados não poderiam ser tomados como uma medida direta da resolução temporal ${ }^{1}$. Para resolver este problema, vários autores sugeriram o uso do ruído branco, já que este se mantém constante (sem alteração da magnitude do espectro) mesmo quando é interrompido abruptamente por um gap. Outros autores buscaram contornar este problema utilizando tempos de início do estímulo mais lentos ou ruído mascarante, quando são usados outros estímulos acústicos que não o ruído branco ${ }^{1,17}$.

Sendo assim, no que se refere aos marcadores dos testes de detecção de gap, diversos estímulos podem ser utilizados (tons puros, ruídos de banda estreita, de banda larga, etc.), bem como várias formas de apresentação destes estímulos (variação de freqüência, intensidade, duração do estímulo, posição do gap, tempo de início do estímulo sonoro, etc.), os quais podem resultar em limiares de detecção de gap muito discrepantes.

Algumas destas diferenças de gap foram explicadas como sendo resultantes do processamento auditivo temporal "intercanais" e "intra-canal". Quando os marcadores que delineiam o gap dividem freqüências características similares, o paradigma é definido como intra-canal. Neste paradigma, a operação temporal executada é a detecção de descontinuidade no canal ativado pelo som. Este fato ocorre, pois a informação sobre a perturbação do estímulo, que constitui o gap, pode ser carregada por qualquer canal perceptual (ou neural) individual representando o espectro do estímulo 9,16,24.

Um canal de freqüência neural consiste em uma série de neurônios hierárquicos restritos, que recebem inputs de uma única célula ciliada interna da cóclea e que estimulam uma coluna de isofreqüência restrita no córtex auditivo. Desta forma, a detecção de gap "intra-canal" corresponde à detecção de uma descontinuidade no padrão de atividade neural ao longo desta via (da cóclea ao córtex) ${ }^{5,16}$. Inúmeras investigações mostraram que marcadores de freqüências semelhantes determinam limiares de gap melhores que marcadores de freqüências diferentes ${ }^{24,25}$.
As operações perceptuais que suportam estas tarefas de detecção (intra e intercanais) são diferentes. Se a sobreposição entre os canais é substancial porque os dois marcadores estimulam o mesmo canal ou canais similares, então o gap pode ser detectado como uma descontinuidade entre representações neurais co-ativadas. Por outro lado, se há uma sobreposição insuficiente entre as representações ativadas, a detecção do gap tem que contar com o tempo relativo de atividade entre o final da atividade neural do primeiro marcador e o início da atividade neural representando o segundo marcador. Para este último caso, o tempo relativo para percepção da atividade neural é mais longo e resulta em limiares de detecção de gaps mais pobres ${ }^{1,5,24-26}$.

Um segundo paradigma de detecção de gap é o "intercanais", no qual os marcadores são compostos por diferentes freqüências ou orelhas estimuladas ou localização em campo livre. Os limiares são, normalmente, mais altos para este paradigma. Alguns autores sugeriram que os limiares mais altos de gap para o processamento auditivo "intercanais" refletiriam a sobreposição central mais pobre dos canais para os marcadores delimitando o gap ${ }^{1,5,24-26}$.

Além disso, contrariamente ao paradigma "intracanal", que é baseado na percepção da descontinuidade do sinal, o paradigma "intercanais" envolveria a divisão da atenção auditiva. Por este motivo, foi sugerido que o processamento "intercanais" seria de origem mais central, enquanto o processamento "intra-canal" teria origem periférica ${ }^{5}$.

No entanto, outros autores questionaram esta afirmação. As tarefas "intercanais" dependem primariamente de um processamento "intra-canal" e a base temporal para a tarefa "intra-canal" também é dependente das respostas dos neurônios corticais ${ }^{16}$. Da mesma forma, postularam que o córtex auditivo primário teria um papel central na análise requerida para detecção de descontinuidade "intra" e "intercanais" 24,25.

Acrescentou-se, ainda, que gaps introduzidos em ruído de banda larga medem a resolução temporal de uma forma simples e conveniente e que, embora o ruído de banda larga excite muitos canais de diferentes freqüências no sistema auditivo, assume-se que esta tarefa dependa de um processo "intra-canal", ao invés de uma tarefa de comparação entre os canais. Neste caso, o gap ocorre simultaneamente em todos os canais e esta informação é combinada entre os canais em níveis mais altos da via auditiva ${ }^{1,5,24}$. 


\begin{tabular}{|c|c|c|c|c|c|c|c|c|}
\hline \multirow{2}{*}{ Autor } & \multirow{2}{*}{ Ano } & \multirow{2}{*}{ Limiar } & \multicolumn{3}{|c|}{ Marcadores } & \multirow{2}{*}{$\begin{array}{l}\text { Intra- } \\
\text { canal }\end{array}$} & \multirow{2}{*}{$\begin{array}{l}\text { Inter- } \\
\text { canais }\end{array}$} & \multirow{2}{*}{ Outros } \\
\hline & & & Freq. & Intens. & Duração & & & \\
\hline Plomp 20 & 1964 & $2-4 \mathrm{~ms}$ & WN & $\begin{array}{l}25 \text { a } 65 \\
d B N S\end{array}$ & $200 \mathrm{~ms}$ & $x$ & & --- \\
\hline Phillips et al ${ }^{9}$ & 1997 & $5-45 \mathrm{~ms}$ & $\begin{array}{l}\mathrm{NB}(0,3 \mathrm{a} \\
8,5 \mathrm{kHz})\end{array}$ & $40 \mathrm{~dB} N S$ & $600 \mathrm{~ms}$ & $x$ & $x$ & --- \\
\hline He et al ${ }^{21}$ & 1999 & $3,46-4,14 \mathrm{~ms}$ & $\begin{array}{l}\text { BB (com } \\
\text { passa-baixo } \\
\text { em } 5 \mathrm{kHz} \text { ) }\end{array}$ & 70 dB NPS & $\begin{array}{c}100 \mathrm{ou} \\
400 \mathrm{~ms} \\
\text { (gaps posicionados } \\
\text { randomicamente ou } \\
\text { fixos) }\end{array}$ & $x$ & & -- \\
\hline $\begin{array}{c}\text { Heinrich et } \\
\mathrm{al}^{24}\end{array}$ & 2004 & $1,13-10,9 \mathrm{~ms}$ & $\begin{array}{l}\text { Tons puros } \\
\text { (1 e } 2 \mathrm{kHz} \text { ) }\end{array}$ & $70 \mathrm{~dB}$ NPS & $20 \mathrm{~ms}$ & $x$ & $x$ & --- \\
\hline $\begin{array}{l}\text { Roberts e } \\
\text { Lister }^{29}\end{array}$ & 2004 & $2,5-7,8 \mathrm{~ms}$ & BB & $35 \mathrm{~dB} N S$ & $\begin{array}{l}\text { Variáveis (gaps } \\
\text { posicionados } \\
\text { randomicamente) }\end{array}$ & $x$ & $x$ & --- \\
\hline Musiek et $\mathrm{al}^{27}$ & 2005 & $4,9 \mathrm{~ms}$ & WN & $50 \mathrm{~dB} N S$ & $\begin{array}{l}\text { Variáveis (gaps } \\
\text { posicionados } \\
\text { randomicamente) }\end{array}$ & $x$ & & $\begin{array}{l}\text { Teste } \\
\text { GIN }\end{array}$ \\
\hline $\begin{array}{l}\text { Samelli e } \\
\text { Schochat }^{6}\end{array}$ & 2008 & $4,19 \mathrm{~ms}$ & WN & $50 \mathrm{~dB} N S$ & $\begin{array}{l}\text { Variáveis (gaps } \\
\text { posicionados } \\
\text { randomicamente) }\end{array}$ & $x$ & & $\begin{array}{l}\text { Teste } \\
\text { GIN }\end{array}$ \\
\hline Zaidan et al ${ }^{30}$ & 2008 & $\begin{array}{c}4,45 \mathrm{~ms} \text { e } 5,61 \\
\mathrm{~ms}\end{array}$ & WN & $50 \mathrm{~dB} N S$ & $\begin{array}{l}\text { Variáveis (gaps } \\
\text { posicionados } \\
\text { randomicamente) }\end{array}$ & $x$ & & $\begin{array}{l}\text { Teste } \\
\text { GIN }\end{array}$ \\
\hline
\end{tabular}

Legenda: NB - narrow band (ruído de banda estreita); BB - broad band (ruído de banda larga); WN - white noise (ruído branco).

Figura 1 - Trabalhos que investigaram limiares de detecção de gap em jovens adultos com audição dentro dos limites de normalidade

Como forma de enfatizar a variabilidade de limiares de detecção de gap na literatura foram tabulados alguns resultados obtidos em sujeitos com audição dentro dos limites de normalidade (Figura 1).

As pesquisas relacionadas na Figura 1 exemplificam a grande variabilidade existente entre os limiares de detecção de gap. Com base nesta variabilidade, alguns autores propuseram que, provavelmente, as diferenças de limiares são decorrentes dos parâmetros aplicados em cada uma das investigações ${ }^{9}$.

Contudo, existe certa concordância de que, para ruído branco, os limiares de detecção de gap são em torno de 2 a $3 \mathrm{~ms} \mathrm{1,} \mathrm{porém} \mathrm{para} \mathrm{testes} \mathrm{que}$ utilizam condição randômica de posicionamento dos gaps além do ruído branco, os limiares sobem para 4 a $5 \mathrm{~ms}$, conforme observado em alguns estudos $6,21,27,28$.

\section{COMENTÁRIOS FINAIS}

De acordo com o comentado anteriormente, pôde-se notar a importância da resolução temporal, uma vez que esta se constitui num pré-requisito para as habilidades lingüísticas, bem como para a leitura.

Desta forma, instrumentos que avaliem esta habilidade auditiva são fundamentais na prática clínica e, para que possam ser usados, necessitam de normatização quanto aos resultados esperados. Para que isto ocorra, uma ferramenta comum deve ser utilizada, já que variáveis como estímulos e formas de apresentação destes estímulos (freqüência, intensidade, duração do estímulo, posição do gap, tempo de início do estímulo sonoro, etc.) podem resultar em limiares de detecção de gap muito discrepantes, o que poderia comprometer a análise dos dados obtidos com este tipo de avaliação. 


\section{ABSTRACT}

Background: temporal auditory processing and temporal resolution. Purpose: promote a theoretical approach on auditory processing, temporal resolution, and different parameters of markers used at various gap detection tests and how they can interfere in threshold determination. Conclusion: auditory processing and temporal resolution are key-factors for language development. The diverse parameters that can be used in the study of gap detection thresholds can result in quite discrepant thresholds.

KEYWORDS: Auditory Perception; Psychoacoustics; Acoustic Stimulation

\section{REFERÊNCIAS}

1. Moore BCJ. An introduction to the psychology of hearing. 5. ed. San Diego: Academic Press; 2003. 373p.

2. Phillips DP. Neural representation of stimulus times in the primary auditory cortex. Ann N Y Acad Sci. 1993; 682(1):104-18.

3. Buonomano DV, Karmarkar UR. How do we tell time? Neuroscientist. 2002; 8(1):42-51.

4. Shinn JB. Temporal processing: the basics. Hear J. 2003; 56(7):52.

5. Smith NA, Trainor LJ, Shore DI. The development of temporal resolution: between-channel gap detection in infants and adults. J Speech Lang Hear Res. 2006; 49(5):1104-13.

6. Samelli AG, Schochat E. The gaps-in-noise test: gap detection thresholds in normal-hearing young adults. Int J Audiol. 2008; 47(5):238-45

7. Shibata T, Sakashita T, Yamane H, Hashimoto C. Temporal resolution and speech recognition ability of patients with retrocochlear auditory dysfunction. Acta Otolaryngol Suppl. 2004; (554):30-4.

8. Mulsow J, Reichmuth C. Electrophysiological assessment of temporal resolution in pinnipeds. Aquatic Mammals. 2007; 33(1):122-31.

9. Phillips DP, Taylor TL, Hall SE, Carr MM, Mossop JE. Detection of silent intervals between noises activating different perceptual channels: some properties of "central" auditory gap detection. J Acoust Soc Am. 1997; 101(6):3694-705.

10. Eggermont JJ. Firing rate and firing synchrony distinguish dynamic from steady state sound. Neuroreport. 1997; 8(12):2709-13.

11. Muniz LF, Roazzi A, Schochat E, Teixeira CF, Lucena JA. Avaliação da habilidade de resolução temporal, com uso do tom puro, em crianças com e sem desvio fonológico. Rev CEFAC. 2007; 9(4):550-62.

12. Baran JA, Musiek FE. Avaliação comportamental do sistema nervoso auditivo central. In: Musiek FE, Rintelmann WF, organizador. Perspectivas atuais em avaliação auditiva. São Paulo: Manole; 2001. p. 371-409.

13. Watson CS. Temporal acuity and the identification of temporal order: related, but distinct, auditory abilities. Semin Hear. 2004; 25(3):219-27.

14. Divenyi PL. The times of Ira Hirsh: multiple ranges of auditory temporal perception. Semin Hear. 2004; 25(3):229-39.

15. Lister JJ, Roberts RA, Shackelford J, Rogers $\mathrm{CL}$. An adaptive clinical test of temporal resolution. Am J Audiol. 2006; 15(2):133-40.

16. Eggermont JJ. Neural responses in primary auditory cortex mimic psychophysical, acrossfrequency-channel, gap-detection thresholds. J Neurophysiol. 2000 Sep; 84(3):1453-63.

17. Oxenham AJ. Influence of spatial and temporal coding on auditory gap detection. J Acoust Soc Am. 2000; 107(4):2215-23.

18. Rupp A, Gutschalk A, Uppenkamp S, Scherg M. Middle latency auditory evoked fields reflect psychoacoustic gap detection thresholds in human listeners. J Neurophysiol. 2004; 92(4):2239-47.

19. Peiffer AM, Friedman JT, Rosen GD, Fitch RH. Impaired gap detection in juvenile microgyric rats. Brain Res Dev Brain Res. 2004; 152(2):93-8.

20. Plomp R. Rate of decay auditory sensation. J Acoust Soc Am. 1964; 36(2):277-82.

21. He NJ, Horwitz AR, Dubno JR, Mills JH. Psychometric functions for gap detection in noise measured from young and aged subjects. J Acoust Soc Am. 1999; 106(2):966-78.

22. Healy EW, Bacon SP. Effect of spectral frequency range and separation on the perception of asynchronous speech. J Acoust Soc Am. 2007; 121(3):1691-700.

23. Wang J, Fenga Y, Yin S. The effect of gapmarker spectrum on gap-evoked auditory response from the inferior colliculus and auditory cortex of guinea pigs. Int J Audiol. 2006; 45(9):521-7.

24. Heinrich A, Alain C, Schneider BA. Within- and between-channel gap detection in the human auditory cortex. Neuroreport. 2004 Sep; 15(13):2051-6. 
25. Elangovan S, Stuart A. Natural boundaries in gap detection are related to categorical perception of stop consonants. Ear Hear. 2008; 29(5):761-74. 26. Carmichael ME, Hall SE, Phillips DP. Ear and contralateral masker effects on auditory temporal gap detection thresholds. Hear Res. 2008 (in press).

27. Musiek FE, Shinn JB, Jirsa R, Bamiou DE, Baran JA, Zaidan E. GIN (gaps in noise) test performance in subjects with confirmed central auditory nervous system involvement. Ear Hear. 2005; 26(6):608-18. 28. Musiek FE, Zaidan EP, Baran JA, Shinn JB,
Jirsa RE. Assessing temporal processes in adults with LD: the GIN test. Proceedings of the 2004 Convention Of American Academy of Audiology, Salt Lake City; march-april 2004. p. 203.

29. Roberts RA, Lister JJ. Effects of age and hearing loss on gap detection and the precedence effect: broadband stimuli. J Speech Lang Hear Res. 2004; 47(5):965-78.

30. Zaidan E, Garcia AP, Tedesco MLF, Baran JA. Desempenho de adultos jovens

normais em dois testes de resolução temporal. PróFono. 2008; 20(1):19-24.

RECEBIDO EM: 25/08/2007

ACEITO EM: 07/02/2008

Endereço para correspondência:

Rua Cipotânea, 51

Cidade Universitária

São Paulo - SP

CEP: $05360-000$

Tel: (11) 3091-8442

E-mail: alesamelli@usp.br; alesamelli@uol.com.br 